\title{
Solitons in Nonlinear Directional Couplers with Optical Metamaterials by Trial Function Scheme
}

\author{
A.H. Arnous ${ }^{a}$, M. Ekici $^{b}$, S.P. MoshokoA ${ }^{c}$, M. Zaka Ullah ${ }^{d}$, A. Biswas ${ }^{c}$, And M. BeliC $^{e}$ \\ ${ }^{a}$ Department of Physics and Engineering Mathematics, Higher Institute of Engineering, El-Shorouk, Cairo, Egypt \\ ${ }^{b}$ Department of Mathematics, Faculty of Science and Arts, Bozok University, 66100 Yozgat, Turkey \\ ${ }^{c}$ Department of Mathematics and Statistics, Tshwane University of Technology, Pretoria-0008, South Africa \\ ${ }^{d}$ Operator Theory and Applications Research Group, Department of Mathematics, Faculty of Science, King \\ Abdulaziz University, P.O. Box-80203, Jeddah-21589, Saudi Arabia \\ ${ }^{e}$ Science Program, Texas A\&M University at Qatar, P.O. Box 23874, Doha, Qatar
}

(Received March 23, 2017)

This paper obtains soliton solutions to nonlinear directional couplers in optical metamaterials by the aid of trial function method. Three types of couplers are studied. Four forms of nonlinearity are considered. Bright, dark, and singular soliton solutions are retrieved. These soliton solutions appear with certain constraint conditions that guarantee their existence.

DOI: 10.12693/APhysPolA.132.1399

PACS/topics: 42.81.Dp, 42.65.Tg

\section{Introduction}

Optical solitons are key elements in fiber-optic communication across trans-continental and trans-oceanic distances [1-20]. The dynamics of these soliton molecules are present in various optical devices such as optical fibers, crystals, metamaterials and metasurfaces, DWDM systems, couplers, magneto-optic waveguides and several others. This paper will address these solitons propagating through couplers that are with optical metamaterials. The purpose of this paper is to extract exact soliton solutions in such couplers. There are several integration schemes that are utilized these days to secure soliton solutions in such variety of optical devices. These vary from the Riccati equation method, the Lie symmetry analysis, inverse scattering tranform (IST), the Kudryashov method, tanh-coth method, $G^{\prime} / G$-expansion scheme and several others. It must be noted that not all of these schemes are equally powerful. For example, besides IST all the remaining methods fail to retrieve soliton radiation. But on the other hand IST turns out to be an epic failure with power-law, dual-power law, log-law and various other laws of nonlinearity. Thus, all of the integration schemes stand on an equal footing to retrieve soliton solutions to nonlinear evolution equations (NLEEs).

This paper will adopt trial equation scheme to secure bright, dark and singular soliton solutions in couplers. This problem has been, however, studied in the past using the method of undetermined coefficients [19]. There are three types of couplers studied in this paper. Each of these couplers are with four forms of nonlinearity. They are Kerr law, power law, parabolic law and dual-power law. The subsequent section recapitulates the integration

*corresponding author; e-mail: biswas.anjan@gmail.com scheme in a succinct manner followed by its detailed application to obtain the soliton solutions. These solutions come with restrictive conditions known as constraints, for their existence, and these are also presented.

\section{Trial solution method (a brief overview)}

In this section we outline the main steps of the trial equation method as following [6]:

Step-1: We consider the following NLEE for a function

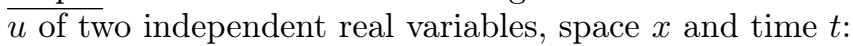

$$
P\left(u, u_{t}, u_{x}, u_{t t}, u_{x x}, u_{x t}, \ldots\right)=0 .
$$

With traveling wave hypothesis

$$
u(x, t)=u(\xi), \quad \xi=k(x-c t),
$$

where $k, c$ are constants to be determined, we reduce Eq. (1) to a nonlinear ordinary differential equation of the form:

$$
F\left(u, u^{\prime}, u^{\prime \prime}, \ldots\right)=0,
$$

where the prime' denotes the derivative $\frac{\mathrm{d}}{\mathrm{d} \xi}$.

Step-2: Take the trial equation

$$
\left(u^{\prime}\right)^{2}=F(u)=\sum_{l=0}^{s} a_{l} u^{l},
$$

where $s$ and $a_{l}$ are constants to be determined. Substituting Eq. (4) and other derivative terms such as $u^{\prime \prime}$ or $u^{\prime \prime \prime}$ and so on into Eq. (3) yields a polynomial $G(u)$ of $u$. According to the balance principle we can determine the value of $s$. Setting the coefficients of $G(u)$ to zero, we get a system of algebraic equations. Solving this system, we shall determine $c, k$ and values of $a_{0}, a_{1}, \ldots, a_{s}$.

Step-3: Rewrite Eq. (4) by the integral form

$$
\pm\left(\xi-\xi_{0}\right)=\int \frac{1}{\sqrt{F(u)}} \mathrm{d} u \text {. }
$$

Based on structure of the polynomial, we classify the roots of $F(u)$, and solve the integral equation (5). Thus we obtain the exact solutions to Eq. (1). 


\section{Twin core couplers}

The governing system of equations for twin-core couplers in optical metamaterials is given by $[19,20]$ :

$$
\begin{aligned}
& \mathrm{i} q_{t}+a_{1} q_{x x}+F\left(|q|^{2}\right) q=\xi_{1}\left(|q|^{2} q\right)_{x x}+\eta_{1}|q|^{2} q_{x x} \\
& \quad+\zeta_{1} q^{2} q_{x x}^{*}+k_{1} r \\
& \mathrm{i} r_{t}+a_{2} r_{x x}+F\left(|r|^{2}\right) r=\xi_{2}\left(|r|^{2} r\right)_{x x}+\eta_{2}|r|^{2} r_{x x} \\
& \quad+\zeta_{2} r^{2} r_{x x}^{*}+k_{2} q .
\end{aligned}
$$

To study the integrability aspects of the governing equations for directional couplers, the following solution structure is taken into consideration:

$$
\begin{aligned}
& q(x, t)=P_{1}(\xi) \mathrm{e}^{\mathrm{i} \Phi(x, t)}, \\
& r(x, t)=P_{2}(\xi) \mathrm{e}^{\mathrm{i} \Phi(x, t)},
\end{aligned}
$$

where the wave variable $\xi$ is given by

$$
\xi=k(x-v t) \text {. }
$$

Here, $P_{l}(\xi)(l=1,2)$ represents the amplitude component of the soliton solutions and $v$ is the speed of the soliton, while the phase component $\Phi(x, t)$ is defined as

$$
\Phi(x, t)=-\kappa x+\omega t+\theta,
$$

where $\kappa$ is the frequency of the solitons, while $\omega$ represents the wave number, and $\theta$ is the phase constant. Substituting (8) and (9) into (6) and (7) and then decomposing into real and imaginary parts gives

$$
\begin{aligned}
& a_{l} k^{2} P_{l}^{\prime \prime}-\left(\omega+a_{l} \kappa^{2}\right)+F\left(P_{l}^{2}\right) P_{l} \\
& +\left(\xi_{l}+\eta_{l}+\zeta_{l}\right) \kappa^{2} P_{l}^{3}-6 \xi_{l} k^{2} P_{l} P_{l}^{\prime 2} \\
& \quad-k^{2}\left(3 \xi_{l}+\eta_{l}+\zeta_{l}\right) P_{l}^{2} P_{l}^{\prime \prime}-k_{l} P_{\tilde{l}}=0,
\end{aligned}
$$

and

$$
\begin{aligned}
& -k v P_{l}^{\prime}-2 a_{l} \kappa k P_{l}^{\prime} \\
& \quad+2 \kappa k^{2}\left(3 \xi_{l}+\eta_{l}-\zeta_{l}\right) P_{l}^{2} P_{l}^{\prime \prime}=0,
\end{aligned}
$$

respectively. Here, $\tilde{l}=3-l$ with $l=1,2$. From the imaginary part Eq. (13), it is possible to obtain the speed of the soliton in each of the waveguide as

$$
v=-2 a_{l} \kappa,
$$

whenever

$$
3 \xi_{l}+\eta_{l}-\zeta_{l}=0
$$

holds. Now, equating the two values of the soliton speed (14) leads to

$$
a_{1}=a_{2}=a .
$$

Consequently, the speed is rewritten as

$$
v=-2 a \kappa,
$$

the coupled NLSE (6)-(7) becomes

$$
\begin{aligned}
& \mathrm{i} q_{t}+a q_{x x}+F\left(|q|^{2}\right) q=\xi_{1}\left(|q|^{2} q\right)_{x x}+\eta_{1}|q|^{2} q_{x x} \\
& \quad+\zeta_{1} q^{2} q^{*}{ }_{x x}+k_{1} r \\
& \mathrm{i} r_{t}+a r_{x x}+F\left(|r|^{2}\right) r=\xi_{2}\left(|r|^{2} r\right)_{x x}+\eta_{2}|r|^{2} r_{x x} \\
& \quad+\zeta_{2} r^{2} r^{*}{ }_{x x}+k_{2} q .
\end{aligned}
$$

and the corresponding modified real part takes the form

$$
\begin{gathered}
a k^{2} P_{l}^{\prime \prime}-\left(\omega+a_{l} \kappa^{2}\right)+F\left(P_{l}^{2}\right) P_{l}+2\left(\zeta_{l}-\xi_{l}\right) \kappa^{2} P_{l}^{3} \\
-6 \xi_{l} k^{2} P_{l}{P_{l}^{\prime}}^{2}-2 k^{2} \zeta_{l} P_{l}^{2} P_{l}^{\prime \prime}-k_{l} P_{\tilde{l}}=0,
\end{gathered}
$$

Using the balancing principle leads to

$$
P_{\tilde{l}}=P_{l} \text {. }
$$

Consequently, Eq. (21) reduces to

$$
\begin{aligned}
& a k^{2} P_{l}^{\prime \prime}-G\left(\omega+a_{l} \kappa^{2}+k_{l}\right)+F\left(P_{l}^{2}\right) P_{l} \\
& \quad+2\left(\zeta_{l}-\xi_{l}\right) \kappa^{2} P_{l}^{3}-6 \xi_{l} k^{2} P_{l}{P_{l}^{\prime}}^{2}-2 k^{2} \zeta_{l} P_{l}^{2} P_{l}^{\prime \prime}=0,
\end{aligned}
$$

In the following subsections, this equation will be studied for four different types of nonlinearity.

\subsection{Kerr law}

For the Kerr law nonlinearity, $F(s)=b s$. The model Eqs. (18) and (19), for twin-core couplers with Kerr law $[19,20]$ nonlinearity, reduces to

$$
\begin{aligned}
& \mathrm{i} q_{t}+a q_{x x}+b_{1}|q|^{2} q=\xi_{1}\left(|q|^{2} q\right)_{x x}+\eta_{1}|q|^{2} q_{x x} \\
& \quad+\zeta_{1} q^{2} q^{*}{ }_{x x}+k_{1} r \\
& \mathrm{i} r_{t}+a r_{x x}+b_{2}|r|^{2} r=\xi_{2}\left(|r|^{2} r\right)_{x x}+\eta_{2}|r|^{2} r_{x x} \\
& \quad+\zeta_{2} r^{2} r^{*}{ }_{x x}+k_{2} q .
\end{aligned}
$$

and Eq. (22) becomes

$$
\begin{aligned}
& a k^{2} P_{l}^{\prime \prime}-\left(\omega+a_{l} \kappa^{2}+k_{l}\right) P_{l}+\left(b_{l}+2\left(\zeta_{l}-\xi_{l}\right) \kappa^{2}\right) P_{l}^{3} \\
& -6 \xi_{l} k^{2} P_{l}{P_{l}^{\prime}}^{2}-2 k^{2} \zeta_{l} P_{l}^{2} P_{l}^{\prime \prime}=0,
\end{aligned}
$$

Balancing $P_{l}^{\prime \prime}$ with $P_{l}^{3}$ in Eq. (25), then we get $s=4$. Using the solution procedure of the trial equation method, we obtain the system of algebraic equations as follows: $P_{l}^{5}$ coeff.:

$$
-2 \alpha_{4} k^{2}\left(2 \zeta_{l}+3 \xi_{l}\right)=0,
$$

$P_{l}^{4}$ coeff.:

$$
-3 \alpha_{3} k^{2}\left(\zeta_{l}+2 \xi_{l}\right)=0
$$

$P_{l}^{3}$ coeff.:

$$
-2 \alpha_{2} k^{2}\left(\zeta_{l}+3 \xi_{l}\right)+2 a \alpha_{4} k^{2}+b_{l}+2 \kappa^{2}\left(\zeta_{l}-\xi_{l}\right)=0,
$$

$P_{l}^{2}$ coeff.:

$$
-\frac{1}{2} k^{2}\left(2 \alpha_{1}\left(\zeta_{l}+6 \xi_{l}\right)-3 a \alpha_{3}\right)=0,
$$

$P_{l}^{1}$ coeff.:

$$
-a \kappa^{2}-6 \alpha_{0} k^{2} \xi_{l}+a \alpha_{2} k^{2}-k_{l}-\omega=0,
$$

$P_{l}^{0}$ coeff.:

$$
\frac{1}{2} a \alpha_{1} k^{2}=0 .
$$

Solving the above system of algebraic equations, we obtain the following results:

$$
\begin{aligned}
& 2 \zeta_{l}+3 \xi_{l}=0, \quad \alpha_{1}=0, \\
& \alpha_{2}=\frac{a \kappa^{2}+6 \alpha_{0} k^{2} \xi_{l}+k_{l}+\omega}{a k^{2}}, \quad \alpha_{3}=0, \\
& \alpha_{4}=-\frac{a\left(b_{l}-8 \kappa^{2} \xi_{l}\right)-18 \alpha_{0} k^{2} \xi_{l}^{2}-3 \xi_{l}\left(k_{l}+\omega\right)}{2 a^{2} k^{2}} .
\end{aligned}
$$

Substituting these results into Eqs. (4) and (5), we get

$$
\pm\left(\xi-\xi_{0}\right)=\int \frac{\mathrm{d} P_{l}}{\sqrt{\alpha_{0}+\alpha_{2} P_{l}^{2}-\alpha_{4} P_{l}^{4}}},
$$

where $\alpha_{0}$ is an arbitrary real constant. Now, we discuss 
two cases as follows:

Case-1: If we set $\alpha_{0}=0$ in Eq. (33) and integrate with respect to $P_{l}$, we get bright soliton solutions as:

$$
\begin{aligned}
& q(x, t)= \pm B_{1} \operatorname{sech}\left(C_{1}\right) \mathrm{e}^{\mathrm{i} \Phi}, \\
& r(x, t)= \pm B_{2} \operatorname{sech}\left(C_{2}\right) \mathrm{e}^{\mathrm{i} \Phi} .
\end{aligned}
$$

These solutions are valid for

$$
\begin{aligned}
& a\left(a \kappa^{2}+k_{l}+\omega\right)>0, \\
& a\left(b_{l}-8 \kappa^{2} \xi_{l}\right)-3 \xi_{l}\left(k_{l}+\omega\right)>0 .
\end{aligned}
$$

Alternatively, we recover singular solitons

$$
\begin{aligned}
& q(x, t)= \pm B_{1} \operatorname{csch}\left(C_{1}\right) \mathrm{e}^{\mathrm{i} \Phi}, \\
& r(x, t)= \pm B_{2} \operatorname{csch}\left(C_{2}\right) \mathrm{e}^{\mathrm{i} \Phi} .
\end{aligned}
$$

These solutions are valid for

$$
\begin{aligned}
& a\left(a \kappa^{2}+k_{l}+\omega\right)>0, \\
& a\left(b_{l}-8 \kappa^{2} \xi_{l}\right)-3 \xi_{l}\left(k_{l}+\omega\right)<0 .
\end{aligned}
$$

In the above case we put:

$B_{i}=\sqrt{\frac{2 a\left(a \kappa^{2}+k_{i}+\omega\right)}{a\left(b_{i}-8 \kappa^{2} \xi_{i}\right)-3 \xi_{i}\left(k_{i}+\omega\right)}}$,

$C_{i}=\sqrt{\frac{a \kappa^{2}+k_{i}+\omega}{a k^{2}}}\left(k(x+2 a \kappa t)-\xi_{0}\right)$,

and $\Phi=(-\kappa x+\omega t+\theta)$ everywhere in this paper.

Case-2: If we set $\alpha_{0}=-\frac{\left(a \kappa^{2}+k_{l}+\omega\right)^{2}}{2 k^{2}\left(a\left(b_{l}-2 \kappa^{2} \xi_{l}\right)+3 \xi_{l}\left(k_{l}+\omega\right)\right)}$ in Eq. (33) and integrating with respect to $P_{l}$, we get dark solitons

$$
\begin{aligned}
& q(x, t)= \pm B_{1} \tanh \left(C_{1}\right) \mathrm{e}^{\mathrm{i} \Phi}, \\
& r(x, t)= \pm B_{2} \tanh \left(C_{2}\right) \mathrm{e}^{\mathrm{i} \Phi},
\end{aligned}
$$

or a second form of singular solitons

$$
\begin{aligned}
& q(x, t)= \pm B_{1} \operatorname{coth}\left(C_{1}\right) \mathrm{e}^{\mathrm{i} \Phi}, \\
& r(x, t)= \pm B_{2} \operatorname{coth}\left(C_{2}\right) \mathrm{e}^{\mathrm{i} \Phi} .
\end{aligned}
$$

These are valid for

$$
\begin{aligned}
& \left(b_{l}-5 \kappa^{2} \xi_{l}\right)\left(a \kappa^{2}+\omega+k_{l}\right)>0, \\
& a\left(b_{l}-2 \kappa^{2} \xi_{l}\right)+3 \xi_{l}\left(\omega+k_{l}\right)<0 .
\end{aligned}
$$

In the above case we put:

$$
\begin{aligned}
B_{i} & =\sqrt{\frac{a \kappa^{2}+\omega+k_{i}}{b_{i}-5 \kappa^{2} \xi_{i}}}, \\
C_{i} & =\sqrt{\frac{\left(5 \kappa^{2} \xi_{i}-b_{i}\right)\left(a \kappa^{2}+\omega+k_{i}\right)}{2 k^{2}\left(a\left(b_{i}-2 \kappa^{2} \xi_{i}\right)+3 \xi_{i}\left(\omega+k_{i}\right)\right)}}\left(k(x+2 a \kappa t)-\xi_{0}\right) .
\end{aligned}
$$

\subsection{Power law}

For power law nonlinear media, $F(s)=b s^{n}$ where $n$ represents the power law nonlinearity factor. The model Eqs. (18) and (19), for twin-core couplers with power law nonlinearity, reduces to [19]

$$
\begin{aligned}
& \mathrm{i} q_{t}+a q_{x x}+b_{1}|q|^{2 n} q=\xi_{1}\left(|q|^{2} q\right)_{x x}+\eta_{1}|q|^{2} q_{x x} \\
& \quad+\zeta_{1} q^{2} q_{x x}^{*}+k_{1} r, \\
& \mathrm{i} r_{t}+a r_{x x}+b_{2}|r|^{2 n} r=\xi_{2}\left(|r|^{2} r\right)_{x x}+\eta_{2}|r|^{2} r_{x x} \\
& \quad+\zeta_{2} r^{2} r^{*}{ }_{x x}+k_{2} q .
\end{aligned}
$$

and Eq. (22) becomes

$$
\begin{aligned}
& a k^{2} P_{l}^{\prime \prime}-\left(\omega+a_{l} \kappa^{2}+k_{l}\right) P_{l}+b_{l} P_{l}^{2 n+1} \\
& \quad+2\left(\zeta_{l}-\xi_{l}\right) \kappa^{2} P_{l}^{3}-6 \xi_{l} k^{2} P_{l}{P_{l}^{\prime}}^{2}-2 k^{2} \zeta_{l} P_{l}^{2} P_{l}^{\prime \prime}=0,
\end{aligned}
$$

To obtain the analytic solution, the transformations

$$
\zeta_{l}=\xi_{l}=0,
$$

are applied in Eq. (50) and give

$$
a k^{2} P_{l}^{\prime \prime}-\left(\omega+a \kappa^{2}+k_{l}\right) P_{l}+b_{l} P_{l}^{2 n+1}=0 .
$$

Then, in order to obtain closed-form solutions, we use the transformation

$$
P_{l}=\sqrt[n]{U_{l}}
$$

so that (52) transforms to

$$
\begin{aligned}
& a k^{2}\left(n U_{l} U_{l}^{\prime \prime}+(1-n) U_{l}^{\prime 2}\right)-n^{2}\left(a \kappa^{2}+k_{l}+\omega\right) U_{l}^{2} \\
& \quad+n^{2} b_{l} U_{l}^{4}=0 .
\end{aligned}
$$

Balancing $U_{l} U_{l}^{\prime \prime}$ with $U_{l}^{4}$ in Eq. (54), then we get $s=$ 4. Using the solution procedure of the trial equation method, we obtain the system of algebraic equations as follows:

$U_{l}^{4}$ coeff.:

$$
a \alpha_{4} k^{2}(n+1)+n^{2} b_{l}=0,
$$

$U_{l}^{3}$ coeff.:

$$
\frac{1}{2} a \alpha_{3} k^{2}(n+2)=0,
$$

$U_{l}^{2}$ coeff.:

$$
a \alpha_{2} k^{2}-n^{2}\left(a \kappa^{2}+k_{l}+\omega\right)=0,
$$

$U_{l}^{1}$ coeff.:

$$
-\frac{1}{2} a \alpha_{1} k^{2}(n-2)=0,
$$

$U_{l}^{0}$ coeff.:

$$
-a \alpha_{0} k^{2}(n-1)=0 .
$$

Solving the above system of algebraic equations, we obtain the following results:

$$
\begin{aligned}
& \alpha_{0}=0, \quad \alpha_{1}=0, \quad \alpha_{2}=\frac{n^{2}\left(a \kappa^{2}+k_{l}+\omega\right)}{a k^{2}}, \\
& \alpha_{3}=0, \quad \alpha_{4}=-\frac{n^{2} b_{l}}{a k^{2}(n+1)} .
\end{aligned}
$$

Substituting these results into Eqs. (4) and (5), we get

$$
\pm\left(\xi-\xi_{0}\right)=\int \frac{\mathrm{d} U_{l}}{\sqrt{\alpha_{2} U_{l}^{2}-\alpha_{4} U_{l}^{4}}} .
$$

Integrating (61) with respect to $U_{l}$, we obtain bright solitons

$$
\begin{aligned}
& q(x, t)=\sqrt[n]{ \pm B_{1} \operatorname{sech}\left(C_{1}\right)} \mathrm{e}^{\mathrm{i} \Phi}, \\
& r(x, t)=\sqrt[n]{ \pm B_{2} \operatorname{sech}\left(C_{2}\right)} \mathrm{e}^{\mathrm{i} \Phi} .
\end{aligned}
$$

These solutions exist for

$$
\begin{aligned}
& a \kappa^{2}+k_{l}+\omega>0, \\
& a>0, \quad b_{l}>0 .
\end{aligned}
$$

The singular solitons are given by

$$
\begin{aligned}
& q(x, t)=\sqrt[n]{ \pm B_{1} \operatorname{csch}\left(C_{1}\right)} \mathrm{e}^{\mathrm{i} \Phi}, \\
& r(x, t)=\sqrt[n]{ \pm B_{2} \operatorname{csch}\left(C_{2}\right)} \mathrm{e}^{\mathrm{i} \Phi} .
\end{aligned}
$$

These exist whenever

$$
\begin{aligned}
& a \kappa^{2}+k_{l}+\omega>0, \\
& a>0, \quad b_{l}<0 .
\end{aligned}
$$

In the above case we put:

$$
B_{i}=\sqrt{\frac{(n+1)\left(a \kappa^{2}+k_{i}+\omega\right)}{b_{i}}},
$$


$C_{i}=\sqrt{\frac{n^{2}\left(a \kappa^{2}+k_{i}+\omega\right)}{a k^{2}}}\left(k(x+2 a \kappa t)-\xi_{0}\right)$.

\subsection{Parabolic law}

For parabolic law nonlinear media, $F(s)=b s^{2}+c s^{4}$. The model Eqs. (18) and (19), for twin-core couplers with parabolic law nonlinearity, reduces to [19]:

$$
\begin{aligned}
& \mathrm{i} q_{t}+a q_{x x}+\left(b_{1}|q|^{2}+c_{1}|q|^{4}\right) q=\xi_{1}\left(|q|^{2} q\right)_{x x} \\
& \quad+\eta_{1}|q|^{2} q_{x x}+\zeta_{1} q^{2} q^{*}{ }_{x x}+k_{1} r, \\
& \mathrm{i} r_{t}+a r_{x x}+\left(b_{1}|q|^{2}+c_{1}|q|^{4}\right) r=\xi_{2}\left(|r|^{2} r\right)_{x x} \\
& \quad+\eta_{2}|r|^{2} r_{x x}+\zeta_{2} r^{2} r^{*}{ }_{x x}+k_{2} q .
\end{aligned}
$$

and Eq. (22) becomes

$$
\begin{aligned}
& a k^{2} P_{l}^{\prime \prime}-\left(\omega+a_{l} \kappa^{2}+k_{l}\right) P_{l}+\left(b_{l}+2\left(\zeta_{l}-\xi_{l}\right) \kappa^{2}\right) P_{l}^{3} \\
& +c_{l} P_{l}^{5}-6 \xi_{l} k^{2} P_{l}{P_{l}^{\prime}}^{2}-2 k^{2} \zeta_{l} P_{l}^{2} P_{l}^{\prime \prime}=0 .
\end{aligned}
$$

Set

$$
P_{l}=U_{l}^{\frac{1}{2}},
$$

so that (72) transforms to

$$
\begin{gathered}
a k^{2}\left(2 U_{l} U_{l}^{\prime \prime}-U_{l}^{\prime 2}\right)-4\left(a \kappa^{2}+k_{l}+\omega\right) U_{l}^{2} \\
-2 k^{2} \zeta_{l}\left(2 U_{l} U_{l}^{\prime \prime}-U_{l}^{\prime 2}\right) U_{l}-6 k^{2} \xi_{l} U_{l} U_{l}^{\prime 2} \\
+4\left(b_{l}+2\left(\zeta_{l}-\xi_{l}\right)\right) U_{l}^{3}+4 c_{l} U_{l}^{4}=0 .
\end{gathered}
$$

Balancing $U_{l} U_{l}^{\prime \prime}$ with $U_{l}^{4}$ in Eq. (74), then we get $s=$ 4. Using the solution procedure of the trial equation method, we obtain the system of algebraic equations as follows:

$U_{l}^{5}$ coeff.:

$$
-6 \alpha_{4} k^{2}\left(\zeta_{l}+\xi_{l}\right)=0,
$$

$U_{l}^{4}$ coeff.:

$$
-2 \alpha_{3} k^{2}\left(2 \zeta_{l}+3 \xi_{l}\right)+3 a \alpha_{4} k^{2}+4 c_{l}=0,
$$

$U_{l}^{3}$ coeff.:

$$
-2 \alpha_{2} k^{2} \zeta_{l}-6 \alpha_{2} k^{2} \xi_{l}+2 a \alpha_{3} k^{2}+4 b_{l}+8\left(\zeta_{l}-\xi_{l}\right)=0,
$$

$U_{l}^{2}$ coeff.:

$$
a \alpha_{2} k^{2}-2\left(2 a \kappa^{2}+3 \alpha_{1} k^{2} \xi_{l}+2\left(k_{l}+\omega\right)\right)=0,
$$

$U_{l}^{1}$ coeff.:

$$
2 \alpha_{0} k^{2}\left(\zeta_{l}-3 \xi_{l}\right)=0
$$

$U_{l}^{0}$ coeff.:

$$
-a \alpha_{0} k^{2}=0 .
$$

Solving the above system of algebraic equations, we obtain the following results:

$$
\begin{gathered}
\zeta_{l}+\xi_{l}=0, \quad \alpha_{0}=0, \quad \alpha_{2}=\frac{4\left(a \kappa^{2}+k_{l}+\omega\right)}{a k^{2}}, \\
\alpha_{3}=\frac{8 \xi_{l}\left(a \kappa^{2}+a+k_{l}+\omega\right)-2 a b_{l}}{a^{2} k^{2}}, \\
\alpha_{4}=-\frac{4\left(a^{2} c_{l}-\xi_{l}\left(4 \xi_{l}\left(a \kappa^{2}+a+k_{l}+\omega\right)-a b_{l}\right)\right)}{3 a^{3} k^{2}} .
\end{gathered}
$$

Substituting these results into Eqs. (4) and (5), we get

$$
\pm\left(\xi-\xi_{0}\right)=\int \frac{\mathrm{d} U_{l}}{\sqrt{\alpha_{1} U_{l}+\alpha_{2} U_{l}^{2}+\alpha_{3} U_{l}^{3}-\alpha_{4} U_{l}^{4}}} .
$$

where $\alpha_{1}$ is an arbitrary real constant. If we set $\alpha_{1}=0$ in Eq. (82) and integrating with respect to $U_{l}$, we obtain bright solitons

$$
\begin{aligned}
& q(x, t)=\sqrt{\frac{8 a\left(a \kappa^{2}+k_{1}+\omega\right)}{ \pm \sqrt{A_{1}} \cosh \left(B_{1}\right)+C_{1}}} \mathrm{e}^{\mathrm{i} \Phi}, \\
& r(x, t)=\sqrt{\frac{8 a\left(a \kappa^{2}+k_{2}+\omega\right)}{ \pm \sqrt{A_{2}} \cosh \left(B_{2}\right)+C_{2}}} \mathrm{e}^{\mathrm{i} \Phi} .
\end{aligned}
$$

These solitons exist with constraints

$$
a\left(a \kappa^{2}+k_{l}+\omega\right)>0, \quad A_{l}>0 .
$$

The singular solitons are

$$
\begin{aligned}
& q(x, t)=\sqrt{\frac{8 a\left(a \kappa^{2}+k_{1}+\omega\right)}{ \pm \sqrt{-A_{1}} \sinh \left(B_{2}\right)+C_{2}}} \mathrm{e}^{\mathrm{i} \Phi}, \\
& r(x, t)=\sqrt{\frac{8 a\left(a \kappa^{2}+k_{2}+\omega\right)}{ \pm \sqrt{-A_{2}} \sinh \left(B_{2}\right)+C_{2}}} \mathrm{e}^{\mathrm{i} \Phi} .
\end{aligned}
$$

These existence criteria is

$$
a\left(a \kappa^{2}+k_{l}+\omega\right)>0, \quad A_{l}<0 .
$$

In the above case we put: $A_{i}=\frac{64}{3}\left(a \kappa^{2}+k_{i}+\omega\right)$

$$
\begin{aligned}
& \times\left(a^{2} c_{i}-\xi_{i}\left(4 \xi_{i}\left(a \kappa^{2}+a+k_{i}+\omega\right)-a b_{i}\right)\right) \\
& +\left(2 a b_{i}-8 \xi_{i}\left(a \kappa^{2}+a+k_{i}+\omega\right)\right)^{2} . \\
B_{i} & =\sqrt{\frac{4\left(a \kappa^{2}+k_{i}+\omega\right)}{a k^{2}}}\left(k(x+2 a \kappa t)-\xi_{0}\right), \\
C_{i}= & \sqrt{\frac{n^{2}\left(a \kappa^{2}+k_{i}+\omega\right)}{a k^{2}}}\left(k(x+2 a \kappa t)-\xi_{0}\right) .
\end{aligned}
$$

\subsection{Dual power law}

For dual power law nonlinear media, $F(s)=b s^{2 n}+$ $c s^{4 n}$. The model Eqs. (18) and (19), for twin-core couplers with dual power law nonlinearity, reduces to [19]:

$$
\begin{aligned}
& \mathrm{i} q_{t}+a q_{x x}+\left(b_{1}|q|^{2 n}+c_{1}|q|^{4 n}\right) q=\xi_{1}\left(|q|^{2} q\right)_{x x} \\
& \quad+\eta_{1}|q|^{2} q_{x x}+\zeta_{1} q^{2} q^{*}{ }_{x x}+k_{1} r, \\
& \mathrm{i} r_{t}+a r_{x x}+\left(b_{1}|q|^{2 n}+c_{1}|q|^{4 n}\right) r=\xi_{2}\left(|r|^{2} r\right)_{x x} \\
& \quad+\eta_{2}|r|^{2} r_{x x}+\zeta_{2} r^{2} r^{*}{ }_{x x}+k_{2} q,
\end{aligned}
$$

and Eq. (22) becomes

$$
\begin{aligned}
& a k^{2} P_{l}^{\prime \prime}-\left(\omega+a_{l} \kappa^{2}+k_{l}\right) P_{l} \\
& \quad+\left(b_{l}+2\left(\zeta_{l}-\xi_{l}\right) \kappa^{2}\right) P_{l}^{2 n+1}+c_{l} P_{l}^{4 n+1} \\
& \quad-6 \xi_{l} k^{2} P_{l}{P_{l}^{\prime 2}}^{2}-2 k^{2} \zeta_{l} P_{l}^{2} P_{l}^{\prime \prime}=0 .
\end{aligned}
$$

To obtain the analytic solution, the transformations

$$
\zeta_{l}=\xi_{l}=0
$$

are applied in Eq. (92) and give

$$
\begin{aligned}
& a k^{2} P_{l}^{\prime \prime}-\left(\omega+a \kappa^{2}+k_{l}\right) P_{l}+b_{l} P_{l}^{2 n+1} \\
& \quad+c_{l} P_{l}^{4 n+1}=0 .
\end{aligned}
$$

Then, in order to obtain closed-form solutions, we use the transformation

$$
P_{l}=U_{l}^{\frac{1}{2 n}} .
$$




$$
\begin{aligned}
& a k^{2}\left(2 n U_{l} U_{l}^{\prime \prime}+(1-2 n) U_{l}^{\prime 2}\right)-4 n^{2}\left(a \kappa^{2}+k_{l}+\omega\right) U_{l}^{2} \\
& \quad+4 n^{2} b_{l} U_{l}^{3}+4 n^{2} c_{l} U_{l}^{4}=0 .
\end{aligned}
$$

Balancing $U_{l} U_{l}^{\prime \prime}$ with $U_{l}^{4}$ in Eq. (96), then we get $s=$ 4. Using the solution procedure of the trial equation method, we obtain the system of algebraic equations as follows:

$U_{l}^{4}$ coeff.:

$$
a \alpha_{4} k^{2}(2 n+1)+4 n^{2} c_{l}=0,
$$

$U_{l}^{3}$ coeff.:

$$
a \alpha_{3} k^{2}(n+1)+4 n^{2} b_{l}=0,
$$

$U_{l}^{2}$ coeff.:

$$
a \alpha_{2} k^{2}-4 n^{2}\left(a \kappa^{2}+k_{l}+\omega\right)=0,
$$

$U_{l}^{1}$ coeff.:

$$
-a \alpha_{1} k^{2}(n-1)=0,
$$

$U_{l}^{0}$ coeff.:

$$
a \alpha_{0} k^{2}(1-2 n)=0,
$$

Solving the above system of algebraic equations, we obtain the following results:

$$
\begin{aligned}
& \alpha_{0}=0, \quad \alpha_{1}=0, \quad \alpha_{2}=\frac{4 n^{2}\left(a \kappa^{2}+k_{l}+\omega\right)}{a k^{2}}, \\
& \alpha_{3}=-\frac{4 n^{2} b_{l}}{a k^{2}(n+1)}, \quad \alpha_{4}=-\frac{4 n^{2} c_{l}}{a k^{2}(2 n+1)} .
\end{aligned}
$$

Substituting these results into Eqs. (4) and (5), we get

$$
\pm\left(\xi-\xi_{0}\right)=\int \frac{\mathrm{d} U_{l}}{\sqrt{\alpha_{2} U_{l}^{2}-\alpha_{3} U_{l}^{3}-\alpha_{4} U_{l}^{4}}} .
$$

Integrating (103) with respect to $U_{l}$, we obtain bright solitons

$$
\begin{aligned}
& q(x, t)=\sqrt[2 n]{\frac{2(n+1)\left(a \kappa^{2}+k_{1}+\omega\right)}{ \pm B_{1} \cosh \left(C_{1}\right)+b_{1}}} \mathrm{e}^{\mathrm{i} \Phi}, \\
& r(x, t)=\sqrt[2 n]{\frac{2(n+1)\left(a \kappa^{2}+k_{2}+\omega\right)}{ \pm B_{2} \cosh \left(C_{2}\right)+b_{2}}} \mathrm{e}^{\mathrm{i} \Phi} .
\end{aligned}
$$

These stay valid when

$$
\begin{aligned}
& a\left(a \kappa^{2}+k_{l}+\omega\right)>0, \\
& 4(n+1)^{2} c_{l}\left(a \kappa^{2}+k_{l}+\omega\right)+(2 n+1) b_{l}^{2}>0 .
\end{aligned}
$$

The singular solitons are given by

$$
\begin{aligned}
& q(x, t)=\sqrt[2 n]{\frac{2(n+1)\left(a \kappa^{2}+k_{1}+\omega\right)}{ \pm B_{1} \sinh \left(C_{1}\right)+b_{1}}} \mathrm{e}^{\mathrm{i} \Phi}, \\
& r(x, t)=\sqrt[2 n]{\frac{2(n+1)\left(a \kappa^{2}+k_{2}+\omega\right)}{ \pm B_{2} \sinh \left(C_{2}\right)+b_{2}}} \mathrm{e}^{\mathrm{i} \Phi} .
\end{aligned}
$$

They exist when

$$
\begin{aligned}
& a\left(a \kappa^{2}+k_{l}+\omega\right)>0, \\
& 4(n+1)^{2} c_{l}\left(a \kappa^{2}+k_{l}+\omega\right)+(2 n+1) b_{l}^{2}<0 .
\end{aligned}
$$

In the above case we put:

$$
\begin{aligned}
& B_{i}=\sqrt{\frac{4 c_{i}(n+1)^{2}\left(a \kappa^{2}+k_{i}+\omega\right)+b_{i}^{2}(2 n+1)}{2 n+1}}, \\
& C_{i}=\sqrt{\frac{4 n^{2}\left(a \kappa^{2}+k_{i}+\omega\right)}{a k^{2}}}\left(k(x+2 a \kappa t)-\xi_{0}\right) .
\end{aligned}
$$

\section{Multiple-core couplers (coupling with nearest neighbors)}

The system describing the dynamics of multiple-core couplers is given by [19]

$$
\begin{aligned}
& \mathrm{i} q_{t}^{(l)}+a_{l} q_{x x}^{(l)}+F\left(\left|q^{(l)}\right|^{2}\right) q^{(l)}=\xi_{l}\left(\left|q^{(l)}\right|^{2} q^{(l)}\right)_{x x} \\
& \quad+\eta_{l}\left|q^{(l)}\right|^{2} q_{x x}^{(l)}+\zeta_{l} q^{(l)^{2}} q^{(l)^{*}}{ }_{x x} \\
& \quad+K\left(q^{(l-1)}-2 q^{(l)}+q^{(l+1)}\right),
\end{aligned}
$$

where $1 \leq l \leq N$. Equation (112) represents the general model for optical couplers where coupling with nearest neighbors is considered. Here, $K$ is, as before, the coupling coefficient. In order to address this model for the five forms of nonlinear media, the initial hypothesis is taken to be

$$
q^{(l)}(x, t)=P_{1}(\xi) \mathrm{e}^{\mathrm{i} \Phi(x, t)},
$$

where $P_{l}(x, t)$ is the amplitude component of the soliton, which carries the same definition as in (8) or (9). After substituting the initial guess (113) into (112), the resulting expression is split into real and imaginary elements. The imaginary part allows one to calculate the speed of the soliton as

$$
v=-2 a_{l} \kappa,
$$

provided that

$$
3 \xi_{l}+\eta_{l}-\zeta_{l}=0,
$$

as was the case for twin couplers. Notice that this speed of the soliton stays the same irrespective of the type of nonlinearity and type of soliton to be considered. Now, for the real part portion, one gets

$$
\begin{aligned}
& a_{l} k^{2} P_{l}^{\prime \prime}-\left(\omega+a_{l} \kappa^{2}\right)+F\left(P_{l}^{2}\right) P_{l}+2\left(\zeta_{l}-\xi_{l}\right) \kappa^{2} P_{l}^{3} \\
& \quad-6 \xi_{l} k^{2} P_{l}{P_{l}^{\prime}}^{2}-2 k^{2} \zeta_{l} P_{l}^{2} P_{l}^{\prime \prime} \\
& -K\left(P_{l-1}-2 P_{l}+P_{l+1}\right)=0,
\end{aligned}
$$

Using the balancing principle leads to

$$
P_{l-1}=P_{l}=P_{l+1},
$$

Consequently, Eq. (116) reduces to

$$
\begin{aligned}
& a_{l} k^{2} P_{l}^{\prime \prime}-\left(\omega+a_{l} \kappa^{2}\right)+F\left(P_{l}^{2}\right) P_{l}+2\left(\zeta_{l}-\xi_{l}\right) \kappa^{2} P_{l}^{3} \\
& -6 \xi_{l} k^{2} P_{l}{P_{l}^{\prime}}^{2}-2 k^{2} \zeta_{l} P_{l}^{2} P_{l}^{\prime \prime}=0 .
\end{aligned}
$$

In the following subsections, this equation will be studied for four different types of nonlinearity.

\subsection{Kerr law}

For the Kerr law nonlinearity, $F(s)=b s$. Eq. (112), for multiple-core couplers (coupling with nearest neighbors) with the Kerr law nonlinearity, reduces to [19]:

$$
\begin{aligned}
& \mathrm{i} q_{t}^{(l)}+a_{l} q_{x x}^{(l)}+b_{l}\left|q^{(l)}\right|^{2} q=\xi_{l}\left(\left|q^{(l)}\right|^{2} q^{(l)}\right)_{x x} \\
& \quad+\eta_{l}\left|q^{(l)}\right|^{2} q_{x x}^{(l)}+\zeta_{l} q^{(l)^{2}} q^{(l)^{*}}{ }_{x x} \\
& +K\left(q^{(l-1)}-2 q^{(l)}+q^{(l+1)}\right),
\end{aligned}
$$

and Eq. (118) becomes

$$
a_{l} k^{2} P_{l}^{\prime \prime}-\left(\omega+a_{l} \kappa^{2}\right)+\left(b_{l}+2\left(\zeta_{l}-\xi_{l}\right) \kappa^{2}\right) P_{l}^{3}
$$




$$
-6 \xi_{l} k^{2} P_{l}{P_{l}^{\prime}}^{2}-2 k^{2} \zeta_{l} P_{l}^{2} P_{l}^{\prime \prime}=0,
$$

Balancing $P_{l}^{\prime \prime}$ with $P_{l}^{3}$ in Eq. (120), then we get $s=$ 4. Using the solution procedure of the trial equation method, we obtain the system of algebraic equations as follows:

$P_{l}^{5}$ coeff.:

$$
-2 \alpha_{4} k^{2}\left(2 \zeta_{l}+3 \xi_{l}\right)=0
$$

$P_{l}^{4}$ coeff.:

$$
-3 \alpha_{3} k^{2}\left(\zeta_{l}+2 \xi_{l}\right)=0,
$$

$P_{l}^{3}$ coeff.

$$
\begin{aligned}
& -2 \alpha_{2} k^{2}\left(\zeta_{l}+3 \xi_{l}\right)+2 a_{l} \alpha_{4} k^{2}+b_{l}+2 \kappa^{2} \zeta_{l} \\
& -2 \kappa^{2} \xi_{l}=0,
\end{aligned}
$$

$P_{l}^{2}$ coeff.:

$$
-\frac{1}{2} k^{2}\left(2 \alpha_{1}\left(\zeta_{l}+6 \xi_{l}\right)-3 a_{l} \alpha_{3}\right)=0
$$

$P_{l}^{1}$ coeff.:

$$
-a_{l} \kappa^{2}-6 \alpha_{0} k^{2} \xi_{l}+a_{l} \alpha_{2} k^{2}-\omega=0,
$$

$P_{l}^{0}$ coeff.:

$$
\frac{1}{2} a_{l} \alpha_{1} k^{2}=0 \text {. }
$$

Solving the above system of algebraic equations, we obtain the following results:

$$
\begin{gathered}
2 \zeta_{l}+3 \xi_{l}=0, \quad \alpha_{1}=0, \quad \alpha_{2}=\frac{a_{l} \kappa^{2}+6 \alpha_{0} k^{2} \xi_{l}+\omega}{a_{l} k^{2}}, \\
\alpha_{3}=0, \quad \alpha_{4}=\frac{\xi_{l}\left(8 a_{l} \kappa^{2}+18 \alpha_{0} k^{2} \xi_{l}+3 \omega\right)-a_{l} b_{l}}{2 a_{l}^{2} k^{2}} .
\end{gathered}
$$

Substituting these results into Eqs. (4) and (5), we get

$$
\pm\left(\xi-\xi_{0}\right)=\int \frac{\mathrm{d} P_{l}}{\sqrt{\alpha_{0}+\alpha_{2} P_{l}^{2}+\alpha_{4} P_{l}^{4}}} .
$$

where $\alpha_{0}$ is an arbitrary real constant. Now, we discuss two cases as follows:

Case-1: If we set $\alpha_{0}=0$ in Eq. (128) and integrating with respect to $P_{l}$, we obtain bright solitons

$$
q^{(l)}(x, t)= \pm B_{l} \operatorname{sech}\left(C_{l}\right) \mathrm{e}^{\mathrm{i} \Phi} .
$$

They exist when

$$
\begin{aligned}
& a_{l}\left(a_{l} \kappa^{2}+\omega\right)>0, \\
& a_{l}\left(b_{l}-8 \kappa^{2} \xi_{l}\right)-3 \xi_{l} \omega>0 .
\end{aligned}
$$

The singular solitons are

$$
q^{(l)}(x, t)= \pm B_{l} \operatorname{csch}\left(C_{l}\right) \mathrm{e}^{\mathrm{i} \Phi} .
$$

This will be valid for

$$
\begin{aligned}
& a_{l}\left(a_{l} \kappa^{2}+\omega\right)>0, \\
& a_{l}\left(b_{l}-8 \kappa^{2} \xi_{l}\right)-3 \xi_{l} \omega<0 .
\end{aligned}
$$

In the above case we put: $B_{l}=\sqrt{\frac{2 a_{l}\left(a_{l} \kappa^{2}+\omega\right)}{a_{l}\left(b_{l}-8 \kappa^{2} \xi_{l}\right)-3 \xi_{l} \omega}}$, $C_{l}=\sqrt{\frac{a_{l} \kappa^{2}+\omega}{a_{l} k^{2}}}\left(k\left(x+2 a_{l} \kappa t\right)-\xi_{0}\right)$.

Case-2: If we set $\alpha_{0}=-\frac{\left(a_{l} \kappa^{2}+\omega\right)^{2}}{2 k^{2}\left(a_{l}\left(b_{l}-2 \kappa^{2} \xi_{l}\right)+3 \xi_{l} \omega\right)}$ in Eq. (128) and integrating with respect to $P_{l}$, we get dark solitons

$$
q^{(l)}(x, t)= \pm B_{l} \tanh \left(C_{l}\right) \mathrm{e}^{\mathrm{i} \Phi},
$$

$$
q^{(l)}(x, t)= \pm B_{l} \operatorname{coth}\left(C_{l}\right) \mathrm{e}^{\mathrm{i} \Phi},
$$

These solutions exist when

$$
\begin{aligned}
& \left(b_{l}-5 \kappa^{2} \xi_{l}\right)\left(a_{l} \kappa^{2}+\omega\right)>0, \\
& a_{l}\left(b_{l}-2 \kappa^{2} \xi_{l}\right)+3 \xi_{l} \omega<0 .
\end{aligned}
$$

In the above case we put: $B_{l}=\sqrt{\frac{a_{l} \kappa^{2}+\omega}{b_{l}-5 \kappa^{2} \xi_{l}}}$,

$C_{l}=\sqrt{-\frac{\left(b_{l}-5 \kappa^{2} \xi_{l}\right)\left(a_{l} \kappa^{2}+\omega\right)}{2 k^{2}\left(a_{l}\left(b_{l}-2 \kappa^{2} \xi_{l}\right)+3 \xi_{l} \omega\right)}}\left(k\left(x+2 a_{l} \kappa t\right)-\xi_{0}\right)$.

\subsection{Power law}

For power law nonlinear media, $F(s)=b s^{n}$ where $n$ represents the power law nonlinearity factor. The model Eq. (112), for twin-core couplers with power law nonlinearity, reduces to [19]

$$
\begin{aligned}
& \mathrm{i} q_{t}^{(l)}+a_{l} q_{x x}^{(l)}+b_{l}\left|q^{(l)}\right|^{2 n} q=\xi_{l}\left(\left|q^{(l)}\right|^{2} q^{(l)}\right)_{x x} \\
& \quad+\eta_{l}\left|q^{(l)}\right|^{2} q_{x x}^{(l)}+\zeta_{l} q^{(l)^{2}} q^{(l)^{*}}{ }_{x x} \\
& \quad+K\left(q^{(l-1)}-2 q^{(l)}+q^{(l+1)}\right),
\end{aligned}
$$

and Eq. (118) becomes

$$
\begin{aligned}
& a_{l} k^{2} P_{l}^{\prime \prime}-\left(\omega+a_{l} \kappa^{2}\right) P_{l}+b_{l} P_{l}^{2 n+1} \\
& +2\left(\zeta_{l}-\xi_{l}\right) \kappa^{2} P_{l}^{3}-6 \xi_{l} k^{2} P_{l}{P_{l}^{\prime 2}}^{2} \\
& -2 k^{2} \zeta_{l} P_{l}^{2} P_{l}^{\prime \prime}=0,
\end{aligned}
$$

To obtain the analytic solution, the transformations

$$
\zeta_{l}=\xi_{l}=0
$$

are applied in Eq. (140) and give

$$
a_{l} k^{2} P_{l}^{\prime \prime}-\left(\omega+a_{l} \kappa^{2}\right) P_{l}+b_{l} P_{l}^{2 n+1}=0 .
$$

Then, in order to obtain closed-form solutions, we use the transformation

$$
P_{l}=U_{l}^{\frac{1}{n}},
$$

so that (142) transforms to

$$
\begin{aligned}
& a_{l} k^{2}\left(n U_{l} U_{l}^{\prime \prime}+(1-n) U_{l}^{\prime 2}\right)-n^{2}\left(a \kappa^{2}+\omega\right) U_{l}^{2} \\
& \quad+n^{2} b_{l} U_{l}^{4}=0 .
\end{aligned}
$$

Balancing $U_{l} U_{l}^{\prime \prime}$ with $U_{l}^{4}$ in Eq. (144), then we get $s=4$. Using the solution procedure of the trial equation method, we obtain the system of algebraic equations as follows:

$U_{l}^{4}$ coeff.:

$$
a_{l} \alpha_{4} k^{2}(n+1)+n^{2} b_{l}=0,
$$

$U_{l}^{3}$ coeff.:

$$
\frac{1}{2} a_{l} \alpha_{3} k^{2}(n+2)=0,
$$

$U_{l}^{2}$ coeff.:

$$
a_{l} \alpha_{2} k^{2}-n^{2}\left(a_{l} \kappa^{2}+\omega\right)=0,
$$

$U_{l}^{1}$ coeff.:

$$
-\frac{1}{2} a_{l} \alpha_{1} k^{2}(n-2)=0,
$$

$U_{l}^{0}$ coeff.:

$$
-a_{l} \alpha_{0} k^{2}(n-1)=0
$$

Solving the above system of algebraic equations, we obtain the following results: 


$$
\begin{aligned}
& \alpha_{0}=0, \quad \alpha_{1}=0, \quad \alpha_{2}=\frac{n^{2}\left(a_{l} \kappa^{2}+\omega\right)}{a_{l} k^{2}}, \\
& \alpha_{3}=0, \quad \alpha_{4}=-\frac{n^{2} b_{l}}{a_{l} k^{2}(n+1)} .
\end{aligned}
$$

Substituting these results into Eqs. (4) and (5), we get

$$
\pm\left(\xi-\xi_{0}\right)=\int \frac{\mathrm{d} U_{l}}{\sqrt{\alpha_{2} U_{l}^{2}-\alpha_{4} U_{l}^{4}}} .
$$

Integrating with respect to $U_{l}$, we obtain bright soliton solution

$$
q^{(l)}(x, t)=\sqrt[n]{ \pm B_{l} \operatorname{sech}\left(C_{l}\right)} \mathrm{e}^{\mathrm{i} \Phi},
$$

This solution is valid for

$$
\begin{aligned}
& a_{l} \kappa^{2}+\omega>0, \\
& a_{l}>0, \quad b_{l}>0 .
\end{aligned}
$$

The singular solitons are given by

$$
q^{(l)}(x, t)=\sqrt[n]{ \pm B_{l} \operatorname{csch}\left(C_{l}\right)} \mathrm{e}^{\mathrm{i} \Phi}
$$

Its domain of existence is

$$
\begin{aligned}
& a_{l} \kappa^{2}+\omega>0, \\
& a_{l}>0, \quad b_{l}>0 .
\end{aligned}
$$

In the above case we put: $B_{l}=\sqrt{\frac{(n+1)\left(a_{l} \kappa^{2}+\omega\right)}{b_{l}}}$, $C_{l}=\sqrt{\frac{n^{2}\left(a_{l} \kappa^{2}+\omega\right)}{a_{l} k^{2}}}\left(k\left(x+2 a_{l} \kappa t\right)-\xi_{0}\right)$.

\subsection{Parabolic law}

For parabolic law nonlinear media, $F(s)=b s^{2}+$ $c s^{4}$. The model Eq. (112), for twin-core couplers with parabolic law nonlinearity, is [19]

$$
\begin{aligned}
& \mathrm{i} q_{t}^{(l)}+a_{l} q_{x x}^{(l)}+\left(b_{l}\left|q^{(l)}\right|^{2}+c_{l}\left|q^{(l)}\right|^{4}\right) q= \\
& \quad \xi_{l}\left(\left|q^{(l)}\right|^{2} q^{(l)}\right)_{x x}+\eta_{l}\left|q^{(l)}\right|^{2} q_{x x}^{(l)}+\zeta_{l} q^{(l)^{2}} q^{(l)}{ }_{x x}^{*} \\
& \quad+K\left(q^{(l-1)}-2 q^{(l)}+q^{(l+1)}\right),
\end{aligned}
$$

and Eq. (118) becomes

$$
\begin{aligned}
& a_{l} k^{2} P_{l}^{\prime \prime}-\left(\omega+a_{l} \kappa^{2}\right) P_{l}+\left(b_{l}+2\left(\zeta_{l}-\xi_{l}\right) \kappa^{2}\right) P_{l}^{3} \\
& +c_{l} P_{l}^{5}-6 \xi_{l} k^{2} P_{l}{P_{l}^{\prime}}^{2}-2 k^{2} \zeta_{l} P_{l}^{2} P_{l}^{\prime \prime}=0 .
\end{aligned}
$$

Set

$$
P_{l}=U_{l}^{\frac{1}{2}}
$$

so that (159) transforms to

$$
\begin{aligned}
& a_{l} k^{2}\left(2 U_{l} U_{l}^{\prime \prime}-U_{l}^{\prime 2}\right)-4\left(a \kappa^{2}+\omega\right) U_{l}^{2} \\
& -2 k^{2} \zeta_{l}\left(2 U_{l} U_{l}^{\prime \prime}-U_{l}^{\prime 2}\right) U_{l}-6 k^{2} \xi_{l} U_{l} U_{l}^{\prime 2} \\
& +4\left(b_{l}+2\left(\zeta_{l}-\xi_{l}\right)\right) U_{l}^{3}+4 c_{l} U_{l}^{4}=0 .
\end{aligned}
$$

Balancing $U_{l} U_{l}^{\prime \prime}$ with $U_{l}^{4}$ in Eq. (161), then we get $s=4$. Using the solution procedure of the trial equation method, we obtain the system of algebraic equations as follows:

$U_{l}^{5}$ coeff.:

$$
-6 \alpha_{4} k^{2}\left(\zeta_{l}+\xi_{l}\right)=0,
$$

$U_{l}^{4}$ coeff.:

$$
-2 \alpha_{3} k^{2}\left(2 \zeta_{l}+3 \xi_{l}\right)+3 a_{l} \alpha_{4} k^{2}+4 c_{l}=0,
$$

$$
\begin{aligned}
& -2 \alpha_{2} k^{2} \zeta_{l}-6 \alpha_{2} k^{2} \xi_{l}+2 a_{l} \alpha_{3} k^{2}+4 b_{l}+8 \zeta_{l} \\
& \quad 8 \xi_{l}=0,
\end{aligned}
$$

$U_{l}^{2}$ coeff.:

$$
a_{l} \alpha_{2} k^{2}-2\left(2 a_{l} \kappa^{2}+3 \alpha_{1} k^{2} \xi_{l}+2 \omega\right)=0,
$$

$U_{l}^{1}$ coeff.:

$$
2 \alpha_{0} k^{2}\left(\zeta_{l}-3 \xi_{l}\right)=0
$$

$U_{l}^{0}$ coeff.:

$$
-a_{l} \alpha_{0} k^{2}=0 .
$$

Solving the above system of algebraic equations, we obtain the following results:

$$
\begin{gathered}
\zeta_{l}+\xi_{l}=0, \quad \alpha_{0}=0, \quad \alpha_{2}=\frac{4\left(a_{l} \kappa^{2}+\omega\right)}{a_{l} k^{2}}, \\
\alpha_{3}=\frac{8 \xi_{l}\left(a_{l} \kappa^{2}+a_{l}+\omega\right)-2 a_{l} b_{l}}{a_{l}^{2} k^{2}}, \\
\alpha_{4}=-\frac{4\left(a_{l}^{2} c_{l}-\xi_{l}\left(4 \xi_{l}\left(a_{l} \kappa^{2}+a_{l}+\omega\right)-a_{l} b_{l}\right)\right)}{3 a_{l}^{3} k^{2}} .
\end{gathered}
$$

Substituting these results into Eqs. (4) and (5), we get

$$
\pm\left(\xi-\xi_{0}\right)=\int \frac{\mathrm{d} U_{l}}{\sqrt{\alpha_{1} U_{l}+\alpha_{2} U_{l}^{2}+\alpha_{3} U_{l}^{3}-\alpha_{4} U_{l}^{4}}}
$$

where $\alpha_{1}$ is an arbitrary real constant. If we set $\alpha_{1}=0$ in Eq. (169) and integrating with respect to $U_{l}$, we obtain bright soliton solution

$$
q^{(l)}(x, t)=\frac{8 a_{l}\left(a_{l} \kappa^{2}+\omega\right)}{ \pm \sqrt{A_{l}} \cosh \left(C_{l}\right)+B_{l}} \mathrm{e}^{\mathrm{i} \Phi} .
$$

They exist with

$$
a_{l}\left(a_{l} \kappa^{2}+\omega\right)>0, \quad A_{l}>0,
$$

The singular solitons are

$$
q(x, t)=\frac{8 a_{1}\left(a_{1} \kappa^{2}+\omega\right)}{ \pm \sqrt{-A_{1}} \sinh \left(C_{l}\right)+B_{l}} \mathrm{e}^{\mathrm{i} \Phi},
$$

Their domain of definition is

$$
a_{l}\left(a_{l} \kappa^{2}+\omega\right)>0, \quad A_{l}<0,
$$

where

$$
\begin{gathered}
A_{l}=\frac{64}{3}\left(a_{l} \kappa^{2}+\omega\right)\left(a_{l}^{2} c_{l}-\xi_{l}\left(4 \xi_{l}\left(a_{l} \kappa^{2}+a_{l}+\omega\right)\right.\right. \\
\left.\left.-a_{l} b_{l}\right)\right)+\left(2 a_{l} b_{l}-8 \xi_{l}\left(a_{l} \kappa^{2}+a_{l}+\omega\right)\right)^{2} .
\end{gathered}
$$

In the above case we put:

$$
\begin{aligned}
& B_{l}=2\left(a_{l} b_{l}-4 \xi_{l}\left(a_{l} \kappa^{2}+a_{l}+\omega\right)\right), \\
& C_{l}=\sqrt{\frac{4\left(a_{l} \kappa^{2}+\omega\right)}{a_{l} k^{2}}}\left(k\left(x+2 a_{l} \kappa t\right)-\xi_{0}\right) .
\end{aligned}
$$

\subsection{Dual power law}

For dual power law nonlinear media, $F(s)=b s^{2 n}+$ $c s^{4 n}$. The model Eq. (112), for twin-core couplers with dual power law nonlinearity, modifies to [19]

$$
\begin{aligned}
& \mathrm{i} q_{t}^{(l)}+a_{l} q_{x x}^{(l)}+\left(b_{l}\left|q^{(l)}\right|^{2 n}+c_{l}\left|q^{(l)}\right|^{4 n}\right) q= \\
& \xi_{l}\left(\left|q^{(l)}\right|^{2} q^{(l)}\right)_{x x}+\eta_{l}\left|q^{(l)}\right|^{2} q_{x x}^{(l)} \\
& +\zeta_{l} q^{(l)^{2}} q_{x x}^{(l)^{*}}+K\left(q^{(l-1)}-2 q^{(l)}+q^{(l+1)}\right),
\end{aligned}
$$

and Eq. (118) becomes

$U_{l}^{3}$ coeff.: 


$$
\begin{aligned}
& a_{l} k^{2} P_{l}^{\prime \prime}-\left(\omega+a_{l} \kappa^{2}\right) P_{l}+\left(b_{l}+2\left(\zeta_{l}-\xi_{l}\right) \kappa^{2}\right) P_{l}^{3} \\
& +c_{l} P_{l}^{5}-6 \xi_{l} k^{2} P_{l}{P_{l}^{\prime}}^{2}-2 k^{2} \zeta_{l} P_{l}^{2} P_{l}^{\prime \prime}=0 .
\end{aligned}
$$

To obtain the analytic solution, the transformations

$$
\zeta_{l}=\xi_{l}=0
$$

are applied in Eq. (176) and give

$$
\begin{aligned}
& a_{l} k^{2} P_{l}^{\prime \prime}-\left(\omega+a_{l} \kappa^{2}\right) P_{l}+b_{l} P_{l}^{2 n+1} \\
& \quad+c_{l} P_{l}^{4 n+1}=0 .
\end{aligned}
$$

Then, in order to obtain closed-form solutions, we use the transformation

$$
P_{l}=U_{l}^{\frac{1}{2 n}} .
$$

so that (178) transforms to

$$
\begin{aligned}
& a_{l} k^{2}\left(2 n U_{l} U_{l}^{\prime \prime}+(1-2 n) U_{l}^{\prime 2}\right)-4 n^{2}\left(a_{l} \kappa^{2}+\omega\right) U_{l}^{2} \\
& \quad+4 n^{2} b_{l} U_{l}^{3}+4 n^{2} c_{l} U_{l}^{4}=0 .
\end{aligned}
$$

Balancing $U_{l} U_{l}^{\prime \prime}$ with $U_{l}^{4}$ in Eq. (180), then we get $s=4$. Using the solution procedure of the trial equation method, we obtain the system of algebraic equations as follows:

$U_{l}^{4}$ coeff.:

$$
a_{l} \alpha_{4} k^{2}(2 n+1)+4 n^{2} c_{l}=0,
$$

$U_{l}^{3}$ coeff.:

$$
a_{l} \alpha_{3} k^{2}(n+1)+4 n^{2} b_{l}=0,
$$

$U_{l}^{2}$ coeff.:

$$
a_{l} a_{2} k^{2}-4 n^{2}\left(a_{l} \kappa^{2}+\omega\right)=0,
$$

$U_{l}^{1}$ coeff.:

$$
-a_{l} \alpha_{1} k^{2}(n-1)=0,
$$

$U_{l}^{0}$ coeff.:

$$
a_{l} \alpha_{0} k^{2}(1-2 n)=0,
$$

Solving the above system of algebraic equations, we obtain the following results:

$$
\begin{aligned}
& \alpha_{0}=0, \quad \alpha_{1}=0, \quad \alpha_{2}=\frac{4 n^{2}\left(a_{l} k^{2}+\omega\right)}{a_{l} k^{2}}, \\
& \alpha_{3}=-\frac{4 n^{2} b_{l}}{a_{l} k^{2}(n+1)}, \quad \alpha_{4}=-\frac{4 n^{2} c_{l}}{a_{l} k^{2}(2 n+1)}
\end{aligned}
$$

Substituting these results into Eqs. (4) and (5), we get

$$
\pm\left(\xi-\xi_{0}\right)=\int \frac{\mathrm{d} U_{l}}{\sqrt{\alpha_{2}} U_{l}^{2}-\alpha_{3} U_{l}^{3}-\alpha_{4} U_{l}^{4}} .
$$

Integrating Eq. (187) with respect to $U_{l}$, we obtain bright solitons

$$
q^{(l)}(x, t)=\sqrt[2 n]{\frac{2(n+1)\left(a_{l} \kappa^{2}+\omega\right)}{ \pm B_{l} \cosh \left(C_{l}\right)+b_{l}}} \mathrm{e}^{\mathrm{i} \Phi},
$$

Their existence criteria is

$$
\begin{aligned}
& a_{l}\left(a_{l} \kappa^{2}+\omega\right)>0, \\
& 4(n+1)^{2} c_{l}\left(a_{l} \kappa^{2}+\omega\right)+(2 n+1) b_{l}^{2}>0 .
\end{aligned}
$$

The singular solitons are:

$$
q^{(l)}(x, t)=\sqrt[2 n]{\frac{2(n+1)\left(a_{l} \kappa^{2}+\omega\right)}{ \pm B_{l} \sinh \left(C_{l}\right)+b_{1}}} \mathrm{e}^{\mathrm{i} \Phi},
$$

These are meaningful whenever

$$
\begin{aligned}
& a_{l}\left(a_{l} \kappa^{2}+\omega\right)>0, \\
& 4(n+1)^{2} c_{l}\left(a_{l} \kappa^{2}+\omega\right)+(2 n+1) b_{l}^{2}<0 .
\end{aligned}
$$

In the above case we put:

$$
\begin{aligned}
& B_{l}=\sqrt{\frac{4 c_{l}(n+1)^{2}\left(a_{l} \kappa^{2}+\omega\right)+b_{l}^{2}(2 n+1)}{2 n+1}}, \\
& C_{l}=\sqrt{\frac{4 n^{2}\left(a_{l} \kappa^{2}+\omega\right)}{a_{l} k^{2}}}\left(k\left(x+2 a_{l} \kappa t\right)-\xi_{0}\right) .
\end{aligned}
$$

\section{Multiple-core couplers (coupling with all neighbors)}

The governing equation that describes the dynamics for multiple couplers, where the coupling action is with all the existing neighbors, is [19]

$$
\begin{gathered}
\mathrm{i} q_{t}^{(l)}+a_{l} q_{x x}^{(l)}+F\left(\left|q^{(l)}\right|^{2}\right) q^{(l)}=\xi_{l}\left(\left|q^{(l)}\right|^{2} q^{(l)}\right)_{x x} \\
+\eta_{l}\left|q^{(l)}\right|^{2} q_{x x}^{(l)}+\zeta_{l} q^{(l)^{2}} q^{(l)^{*}}{ }_{x x}+\sum_{m=1}^{N} \lambda_{l m} q^{(m)},
\end{gathered}
$$

where $1 \leq l \leq N$ while $\lambda_{l m}$ represents the coupling coefficient with all neighbors. The assumption to be considered here is taken to be the same as given by (113). The substitution of this hypothesis into (194) yields the same soliton speed as in (114) subject to (115), which is valid for all nonlinearities and for all the considered solitons. To this end, the real part equation now takes the form

$$
\begin{aligned}
& a_{l} k^{2} P_{l}^{\prime \prime}-\left(\omega+a_{l} \kappa^{2}\right)+F\left(P_{l}^{2}\right) P_{l}+2\left(\zeta_{l}-\xi_{l}\right) \kappa^{2} P_{l}^{3} \\
& \quad-6 \xi_{l} k^{2} P_{l}{P_{l}^{\prime}}^{2}-2 k^{2} \zeta_{l} P_{l}^{2} P_{l}^{\prime \prime} \\
& \quad-\sum_{m=1}^{N} \lambda_{l m} P_{m}=0
\end{aligned}
$$

Using the balancing principle leads to

$$
P_{l}=P_{m},
$$

Consequently, Eq. (195) reduces to

$$
\begin{aligned}
& a_{l} k^{2} P_{l}^{\prime \prime}-\left(\omega+\sum_{m=1}^{N} \lambda_{l m}+a_{l} \kappa^{2}\right)+F\left(P_{l}^{2}\right) P_{l} \\
& \quad+2\left(\zeta_{l}-\xi_{l}\right) \kappa^{2} P_{l}^{3}-6 \xi_{l} k^{2} P_{l}{P_{l}^{\prime}}^{2}-2 k^{2} \zeta_{l} P_{l}^{2} P_{l}^{\prime \prime}=0 .
\end{aligned}
$$

In the following subsections, this equation will be studied for four different types of nonlinearity.

\subsection{Kerr law}

For the Kerr law nonlinearity, $F(s)=b s$. Eq. (194), for multiple-core couplers (coupling with nearest neighbors) with the Kerr law nonlinearity, simplifies to [19]

$$
\begin{gathered}
\mathrm{i} q_{t}^{(l)}+a_{l} q_{x x}^{(l)}+b_{l}\left|q^{(l)}\right|^{2} q=\xi_{l}\left(\left|q^{(l)}\right|^{2} q^{(l)}\right)_{x x} \\
+\eta_{l}\left|q^{(l)}\right|^{2} q_{x x}^{(l)}+\zeta_{l} q^{(l)^{2}} q^{(l)^{*}}{ }_{x x}+\sum_{m=1}^{N} \lambda_{l m},
\end{gathered}
$$

and Eq. (197) becomes

$$
\begin{gathered}
a_{l} k^{2} P_{l}^{\prime \prime}-\left(\omega+\sum_{m=1}^{N} \lambda_{l m}+a_{l} \kappa^{2}\right)-2 k^{2} \zeta_{l} P_{l}^{2} P_{l}^{\prime \prime} \\
+\left(b_{l}+2\left(\zeta_{l}-\xi_{l}\right) \kappa^{2}\right) P_{l}^{3}-6 \xi_{l} k^{2} P_{l}{P_{l}^{\prime}}^{2}=0,
\end{gathered}
$$

Balancing $P_{l}^{\prime \prime}$ with $P_{l}^{3}$ in Eq. (199), then we get $s=$ 
4. Using the solution procedure of the trial equation method, we obtain the system of algebraic equations as follows:

$P_{l}^{5}$ coeff.:

$$
-2 \alpha_{4} k^{2}\left(2 \zeta_{l}+3 \xi_{l}\right)=0
$$

$P_{l}^{4}$ coeff.:

$$
-3 \alpha_{3} k^{2}\left(\zeta_{l}+2 \xi_{l}\right)=0,
$$

$P_{l}^{3}$ coeff.:

$$
\begin{aligned}
& -2 \alpha_{2} k^{2}\left(\zeta_{l}+3 \xi_{l}\right)+2 a_{l} \alpha_{4} k^{2}+b_{l}+2 \kappa^{2} \zeta_{l} \\
& -2 \kappa^{2} \xi_{l}=0,
\end{aligned}
$$

$P_{l}^{2}$ coeff.:

$$
-\frac{1}{2} k^{2}\left(2 \alpha_{1}\left(\zeta_{l}+6 \xi_{l}\right)-3 a_{l} \alpha_{3}\right)=0,
$$

$P_{l}^{1}$ coeff.:

$$
-a_{l} \kappa^{2}-6 \alpha_{0} k^{2} \xi_{l}+a_{l} \alpha_{2} k^{2}-\omega-\sum_{m=1}^{N} \lambda_{l m}=0,
$$

$P_{l}^{0}$ coeff.:

$$
\frac{1}{2} a_{l} \alpha_{1} k^{2}=0 \text {. }
$$

Solving the above system of algebraic equations, we obtain the following results:

$$
\begin{aligned}
& 2 \zeta_{l}+3 \xi_{l}=0, \quad \alpha_{1}=0, \quad \alpha_{3}=0, \\
& \alpha_{2}=\frac{a_{l} \kappa^{2}+6 \alpha_{0} k^{2} \xi_{l}+\omega+\sum_{m=1}^{N} \lambda_{l m}}{a_{l} k^{2}}, \\
& \alpha_{4}=\frac{\xi_{l}\left(8 a_{l} \kappa^{2}+18 \alpha_{0} k^{2} \xi_{l}+3 \omega+3 \sum_{m=1}^{N} \lambda_{l m}\right)-a_{l} b_{l}}{2 a_{l}^{2} k^{2}} .
\end{aligned}
$$

Substituting these results into Eqs. (4) and (5), we get

$$
\pm\left(\xi-\xi_{0}\right)=\int \frac{\mathrm{d} P_{l}}{\sqrt{\alpha_{0}+\alpha_{2} P_{l}^{2}+\alpha_{4} P_{l}^{4}}} .
$$

where $\alpha_{0}$ is an arbitrary real constant. Now, we discuss two cases as follows:

Case-1: If we set $\alpha_{0}=0$ in Eq. (207) and integrating with respect to $P_{l}$, we recover bright soliton solutions

$$
q^{(l)}(x, t)= \pm B_{l} \operatorname{sech}\left(C_{l}\right) \mathrm{e}^{\mathrm{i} \Phi} .
$$

These are valid with

$$
\begin{aligned}
& a_{l}\left(a_{l} \kappa^{2}+\omega+\sum_{m=1}^{N} \lambda_{l m}\right)>0, \\
& a_{l}\left(b_{l}-8 \kappa^{2} \xi_{l}\right)-3 \xi_{l}\left(\omega+\sum_{m=1}^{N} \lambda_{l m}\right)>0 .
\end{aligned}
$$

The singular solitons are given by

$$
q^{(l)}(x, t)= \pm B_{l} \operatorname{csch}\left(C_{l}\right) \mathrm{e}^{\mathrm{i} \Phi} .
$$

These exist when

$$
\begin{aligned}
& a_{l}\left(a_{l} \kappa^{2}+\omega+\sum_{m=1}^{N} \lambda_{l m}\right)>0, \\
& a_{l}\left(b_{l}-8 \kappa^{2} \xi_{l}\right)-3 \xi_{l}\left(\omega+\sum_{m=1}^{N} \lambda_{l m}\right)<0 .
\end{aligned}
$$

In the above case we put:

$$
B_{l}=\sqrt{\frac{2 a_{l}\left(a_{l} \kappa^{2}+\omega+\sum_{m=1}^{N} \lambda_{l m}\right)}{a_{l}\left(b_{l}-8 \kappa^{2} \xi_{l}\right)-3 \xi_{l}\left(\omega+\sum_{m=1}^{N} \lambda_{l m}\right)}},
$$

$C_{l}=\sqrt{\frac{a_{l} \kappa^{2}+\omega+\sum_{m=1}^{N} \lambda_{l m}}{a_{l} k^{2}}}\left(k\left(x+2 a_{l} \kappa t\right)-\xi_{0}\right)$.

Case-2: If we set $\alpha_{0}=$

$\frac{\left(a_{l} \kappa^{2}+\omega+\sum_{m=1}^{N} \lambda_{l m}\right)^{2}}{2 k^{2}\left(a_{l}\left(b_{l}-2 \kappa^{2} \xi_{l}\right)+3 \xi_{l}\left(\omega+\sum_{m=1}^{N} \lambda_{l m}\right)\right)}$ in Eq. (207) and integrating with respect to $P_{l}$, we get dark optical solitons

$$
q^{(l)}(x, t)= \pm B_{l} \tanh \left(C_{l}\right) \mathrm{e}^{\mathrm{i} \Phi},
$$

or singular optical solitons of the second type

$$
q^{(l)}(x, t)= \pm B_{l} \operatorname{coth}\left(C_{l}\right) \mathrm{e}^{\mathrm{i} \Phi},
$$

These solutions are valid for

$$
\begin{aligned}
& \left(b_{l}-5 \kappa^{2} \xi_{l}\right)\left(a_{l} \kappa^{2}+\omega+\sum_{m=1}^{N} \lambda_{l m}\right)>0, \\
& a_{l}\left(b_{l}-2 \kappa^{2} \xi_{l}\right)+3 \xi_{l}\left(\omega+\sum_{m=1}^{N} \lambda_{l m}\right)<0 .
\end{aligned}
$$

In the above case we put:

$$
\begin{aligned}
& B_{l}=\sqrt{\frac{a_{l} \kappa^{2}+\omega+\sum_{m=1}^{N} \lambda_{l m}}{b_{l}-5 \kappa^{2} \xi_{l}}}, \\
& C_{l}=\sqrt{-\frac{\left(b_{l}-5 \kappa^{2} \xi_{l}\right)\left(a_{l} \kappa^{2}+\omega+\sum_{m=1}^{N} \lambda_{l m}\right)}{2 k^{2}\left(a_{l}\left(b_{l}-2 \kappa^{2} \xi_{l}\right)+3 \xi_{l}\left(\omega+\sum_{m=1}^{N} \lambda_{l m}\right)\right)}} \\
& \times\left(k\left(x+2 a_{l} \kappa t\right)-\xi_{0}\right) .
\end{aligned}
$$

\subsection{Power law}

For power law nonlinear media, $F(s)=b s^{n}$. The model Eq. (194), for twin-core couplers with power law nonlinearity, becomes [19]

$$
\begin{gathered}
\mathrm{i} q_{t}^{(l)}+a_{l} q_{x x}^{(l)}+b_{l}\left|q^{(l)}\right|^{2 n} q=\xi_{l}\left(\left|q^{(l)}\right|^{2} q^{(l)}\right)_{x x} \\
+\eta_{l}\left|q^{(l)}\right|^{2} q_{x x}^{(l)}+\zeta_{l} q^{(l)^{2}} q_{x x}^{(l) *}+\sum_{m=1}^{N} \lambda_{l m} q^{(m)},
\end{gathered}
$$

and Eq. (197) becomes

$$
\begin{aligned}
& a_{l} k^{2} P_{l}^{\prime \prime}-\left(\omega+a_{l} \kappa^{2}+\sum_{m=1}^{N} \lambda_{l m}\right) P_{l}+b_{l} P_{l}^{2 n+1} \\
& \quad+2\left(\zeta_{l}-\xi_{l}\right) \kappa^{2} P_{l}^{3}-6 \xi_{l} k^{2} P_{l} P_{l}^{\prime 2} \\
& \quad-2 k^{2} \zeta_{l} P_{l}^{2} P_{l}^{\prime \prime}=0,
\end{aligned}
$$

To obtain the analytic solution, the transformations

$$
\zeta_{l}=\xi_{l}=0,
$$

are applied in Eq. (219) and give

$$
\begin{aligned}
& a_{l} k^{2} P_{l}^{\prime \prime}-\left(\omega+a_{l} \kappa^{2}+\sum_{m=1}^{N} \lambda_{l m}\right) P_{l} \\
& \quad+b_{l} P_{l}^{2 n+1}=0 .
\end{aligned}
$$

Then, in order to obtain closed-form solutions, we use the transformation

$$
P_{l}=U_{l}^{\frac{1}{n}},
$$

so that (221) transforms to

$$
\begin{aligned}
& a_{l} k^{2}\left(n U_{l} U_{l}^{\prime \prime}+(1-n) U_{l}^{\prime 2}\right) \\
& \quad-n^{2}\left(a \kappa^{2}+\sum_{m=1}^{N} \lambda_{l m}+\omega\right) U_{l}^{2}+n^{2} b_{l} U_{l}^{4}=0 .
\end{aligned}
$$

Balancing $U_{l} U_{l}^{\prime \prime}$ with $U_{l}^{4}$ in Eq. (223), then we get $s=4$. Using the solution procedure of the trial equation 
method, we obtain the system of algebraic equations as follows:

$U_{l}^{4}$ coeff.:

$$
a_{l} \alpha_{4} k^{2}(n+1)+n^{2} b_{l}=0,
$$

$U_{l}^{3}$ coeff.:

$$
\frac{1}{2} a_{l} \alpha_{3} k^{2}(n+2)=0,
$$

$U_{l}^{2}$ coeff.:

$$
a_{l} \alpha_{2} k^{2}-n^{2}\left(a_{l} \kappa^{2}+\omega+\sum_{m=1}^{N} \lambda_{l m}\right)=0,
$$

$U_{l}^{1}$ coeff.:

$$
-\frac{1}{2} a_{l} \alpha_{1} k^{2}(n-2)=0
$$

$U_{l}^{0}$ coeff.:

$$
-a_{l} \alpha_{0} k^{2}(n-1)=0 .
$$

Solving the above system of algebraic equations, we obtain the following results:

$$
\begin{aligned}
& \alpha_{0}=0, \quad \alpha_{1}=0, \quad \alpha_{2}=\frac{n^{2}\left(a_{l} \kappa^{2}+\omega+\sum_{m=1}^{N} \lambda_{l m}\right)}{a_{l} k^{2}}, \\
& \alpha_{3}=0, \quad \alpha_{4}=-\frac{n^{2} b_{l}}{a_{l} k^{2}(n+1)} .
\end{aligned}
$$

Substituting these results into Eqs. (4) and (5), we get

$$
\pm\left(\xi-\xi_{0}\right)=\int \frac{\mathrm{d} U_{l}}{\sqrt{\alpha_{2} U_{l}^{2}-\alpha_{4} U_{l}^{4}}} .
$$

integrating with respect to $U_{l}$, we obtain bright soliton solutions

$$
q^{(l)}(x, t)=\sqrt[n]{ \pm B_{l} \operatorname{sech}\left(C_{l}\right)} \mathrm{e}^{\mathrm{i} \Phi}
$$

They are valid for

$$
\begin{aligned}
& a_{l} \kappa^{2}+\omega+\sum_{m=1}^{N} \lambda_{l m}>0, \\
& a_{l}>0, \quad b_{l}>0 .
\end{aligned}
$$

The singular optical solitons are

$$
q^{(l)}(x, t)=\sqrt[n]{ \pm B_{l} \operatorname{csch}\left(C_{l}\right)} \mathrm{e}^{\mathrm{i} \Phi},
$$

These exist when

$$
a_{l} \kappa^{2}+\omega+\sum_{m=1}^{N} \lambda_{l m}>0,
$$$$
a_{l}>0, \quad b_{l}>0 .
$$

In the above case we put:

$$
\begin{aligned}
& B_{l}=\sqrt{\frac{(n+1)\left(a_{l} \kappa^{2}+\omega+\sum_{m=1}^{N} \lambda_{l m}\right)}{b_{l}}}, \\
& C_{l}=\sqrt{\frac{n^{2}\left(a_{l} \kappa^{2}+\omega+\sum_{m=1}^{N} \lambda_{l m}\right)}{a_{l} k^{2}}}\left(\xi-\xi_{0}\right) .
\end{aligned}
$$

\subsection{Parabolic law}

For parabolic law nonlinear media, $F(s)=b s^{2}+$ $c s^{4}$. The model Eq. (194), for twin-core couplers with parabolic law nonlinearity, is [19]

$$
\begin{gathered}
\mathrm{i} q_{t}^{(l)}+a_{l} q_{x x}^{(l)}+\left(b_{l}\left|q^{(l)}\right|^{2}+c_{l}\left|q^{(l)}\right|^{4}\right) q= \\
\xi_{l}\left(\left|q^{(l)}\right|^{2} q^{(l)}\right)_{x x}+\eta_{l}\left|q^{(l)}\right|^{2} q_{x x}^{(l)}
\end{gathered}
$$

$$
+\zeta_{l} q^{(l)^{2}} q^{(l)^{*}}{ }_{x x}+\sum_{m=1}^{N} \lambda_{l m} q^{(m)},
$$

and Eq. (197) becomes

$$
\begin{aligned}
& a_{l} k^{2} P_{l}^{\prime \prime}-\left(\omega+a_{l} \kappa^{2}+\sum_{m=1}^{N} \lambda_{l m}\right) P_{l} \\
& \quad+\left(b_{l}+2\left(\zeta_{l}-\xi_{l}\right) \kappa^{2}\right) P_{l}^{3} \\
& \quad+c_{l} P_{l}^{5}-6 \xi_{l} k^{2} P_{l}{P_{l}^{\prime}}^{2}-2 k^{2} \zeta_{l} P_{l}^{2} P_{l}^{\prime \prime}=0 .
\end{aligned}
$$

Set

$$
P_{l}=U_{l}^{\frac{1}{2}}
$$

so that (238) transforms to

$$
\begin{aligned}
& a_{l} k^{2}\left(2 U_{l} U_{l}^{\prime \prime}-U_{l}^{\prime 2}\right)-4\left(a \kappa^{2}+\sum_{m=1}^{N} \lambda_{l m}+\omega\right) U_{l}^{2} \\
& -2 k^{2} \zeta_{l}\left(2 U_{l} U_{l}^{\prime \prime}-U_{l}^{\prime 2}\right) U_{l}-6 k^{2} \xi_{l} U_{l} U_{l}^{\prime 2} \\
& \quad+4\left(b_{l}+2\left(\zeta_{l}-\xi_{l}\right)\right) U_{l}^{3}+4 c_{l} U_{l}^{4}=0 .
\end{aligned}
$$

Balancing $U_{l} U_{l}^{\prime \prime}$ with $U_{l}^{4}$ in Eq. (167), then we get $s=4$. Using the solution procedure of the trial equation method, we obtain the system of algebraic equations as follows:

$U_{l}^{5}$ coeff.:

$$
-6 \alpha_{4} k^{2}\left(\zeta_{l}+\xi_{l}\right)=0
$$

$U_{l}^{4}$ coeff.:

$$
-2 \alpha_{3} k^{2}\left(2 \zeta_{l}+3 \xi_{l}\right)+3 a_{l} \alpha_{4} k^{2}+4 c_{l}=0,
$$

$U_{l}^{3}$ coeff.:

$$
\begin{aligned}
& -2 \alpha_{2} k^{2} \zeta_{l}-6 \alpha_{2} k^{2} \xi_{l}+2 a_{l} \alpha_{3} k^{2}+4 b_{l}+8 \zeta_{l} \\
& -8 \xi_{l}=0,
\end{aligned}
$$

$U_{l}^{2}$ coeff.:

$$
\begin{gathered}
a_{l} \alpha_{2} k^{2}-4 a_{l} \kappa^{2}+6 \alpha_{1} k^{2} \xi_{l} \\
+4\left(\omega+\sum_{m=1}^{N} \lambda_{l m}\right)=0,
\end{gathered}
$$

$U_{l}^{1}$ coeff.:

$$
2 \alpha_{0} k^{2}\left(\zeta_{l}-3 \xi_{l}\right)=0
$$

$U_{l}^{0}$ coeff.:

$$
-a_{l} \alpha_{0} k^{2}=0 .
$$
tain the following results:

$$
\begin{aligned}
& \zeta_{l}+\xi_{l}=0, \quad \alpha_{0}=0, \\
& \alpha_{2}=\frac{4\left(a_{l} \kappa^{2}+\omega+\sum_{m=1}^{N} \lambda_{l m}\right)}{a_{l} k^{2}}, \\
& \alpha_{3}=\frac{8 \xi_{l}\left(a_{l} \kappa^{2}+a_{l}+\omega+\sum_{m=1}^{N} \lambda_{l m}\right)-2 a_{l} b_{l}}{a_{l}^{2} k^{2}},
\end{aligned}
$$

$\alpha_{4}=-\frac{a_{l}^{2} c_{l}-4 \xi_{l}^{2}\left(a_{l} \kappa^{2}+a_{l}+\omega+\sum_{m=1}^{N} \lambda_{l m}\right)-a_{l} b_{l} \xi_{l}}{0.75 a_{l}^{3} k^{2}}$.

Substituting these results into Eqs. (4) and (5), we get 


$$
\begin{aligned}
& \pm\left(\xi-\xi_{0}\right)= \\
& \quad \int \frac{\mathrm{d} U_{l}}{\sqrt{\alpha_{1} U_{l}+\alpha_{2} U_{l}^{2}+\alpha_{4} U_{l}^{4}}} .
\end{aligned}
$$

where $\alpha_{1}$ is an arbitrary real constant. If we set $\alpha_{1}=0$ in Eq. (248) and integrating with respect to $U_{l}$, we obtain bright solitons

$$
q^{(l)}(x, t)=\frac{8 a_{l}\left(a_{l} \kappa^{2}+\omega+\sum_{m=1}^{N} \lambda_{l m}\right)}{ \pm \sqrt{A_{l}} \cosh \left(C_{l}\right)+B_{l}} \mathrm{e}^{\mathrm{i} \Phi} .
$$

The domain for existence is

$$
a_{l}\left(a_{l} \kappa^{2}+\omega+\sum_{m=1}^{N} \lambda_{l m}\right)>0, \quad A_{l}>0 .
$$

The singular solitons are

$$
q(x, t)=\frac{8 a_{1}\left(a_{1} \kappa^{2}+\omega+\sum_{m=1}^{N} \lambda_{l m}\right)}{ \pm \sqrt{-A_{1}} \sinh \left(C_{l}\right)+B_{l}} \mathrm{e}^{\mathrm{i} \Phi},
$$

These exist with

$$
a_{l}\left(a_{l} \kappa^{2}+\omega+\sum_{m=1}^{N} \lambda_{l m}\right)>0, \quad A_{l}<0
$$

where

$$
\begin{aligned}
& A_{l}=\frac{64}{3}\left(a_{l} \kappa^{2}+\omega+\sum_{m=1}^{N} \lambda_{l m}\right) \\
& \times\left(a_{l}^{2} c_{l}-\xi_{l}\left(4 \xi_{l}\left(a_{l} \kappa^{2}+a_{l}+\omega+\sum_{m=1}^{N} \lambda_{l m}\right)-a_{l} b_{l}\right)\right) \\
&+\left(2 a_{l} b_{l}-8 \xi_{l}\left(a_{l} \kappa^{2}+a_{l}+\omega+\sum_{m=1}^{N} \lambda_{l m}\right)\right)^{2} .(253)
\end{aligned}
$$

In the above case we put:

$$
\begin{aligned}
& B_{l}=2\left(a_{l} b_{l}-4 \xi_{l}\left(a_{l} \kappa^{2}+a_{l}+\omega+\sum_{m=1}^{N} \lambda_{l m}\right)\right), \\
& C_{l}=\sqrt{\frac{4\left(a_{l} \kappa^{2}+\omega+\sum_{m=1}^{N} \lambda_{l m}\right)}{a_{l} k^{2}}}\left(\xi-\xi_{0}\right) .
\end{aligned}
$$

\subsection{Dual power law}

For dual power law nonlinear media, $F(s)=b s^{2 n}+$ $c s^{4 n}$. The model Eq. (194), for twin-core couplers with dual power law nonlinearity, transforms to [19]

$$
\begin{gathered}
\mathrm{i} q_{t}^{(l)}+a_{l} q_{x x}^{(l)}+\left(b_{l}\left|q^{(l)}\right|^{2 n}+c_{l}\left|q^{(l)}\right|^{4 n}\right) q= \\
\xi_{l}\left(\left|q^{(l)}\right|^{2} q^{(l)}\right)_{x x}+\eta_{l}\left|q^{(l)}\right|^{2} q_{x x}^{(l)} \\
+\zeta_{l} q^{(l)^{2}} q^{(l)}{ }_{x x}^{*}+\sum_{m=1}^{N} \lambda_{l m} q^{(m)},
\end{gathered}
$$

and Eq. (197) becomes

$$
\begin{aligned}
& a_{l} k^{2} P_{l}^{\prime \prime}-\left(\omega+a_{l} \kappa^{2}+\sum_{m=1}^{N} \lambda_{l m}\right) P_{l} \\
& \quad+\left(b_{l}+2\left(\zeta_{l}-\xi_{l}\right) \kappa^{2}\right) P_{l}^{3} \\
& \quad+c_{l} P_{l}^{5}-6 \xi_{l} k^{2} P_{l}{P_{l}^{\prime}}^{2}-2 k^{2} \zeta_{l} P_{l}^{2} P_{l}^{\prime \prime}=0 .
\end{aligned}
$$

To obtain the analytic solution, the transformations

$$
\zeta_{l}=\xi_{l}=0
$$

$$
\begin{aligned}
& a_{l} k^{2} P_{l}^{\prime \prime}-\left(\omega+a_{l} \kappa^{2}+\sum_{m=1}^{N} \lambda_{l m}\right) P_{l}+b_{l} P_{l}^{2 n+1} \\
& \quad+c_{l} P_{l}^{4 n+1}=0 .
\end{aligned}
$$

Then, in order to obtain closed-form solutions, we use the transformation

$$
P_{l}=U_{l}^{\frac{1}{2 n}} \text {. }
$$

so that (257) transforms to

$$
\begin{aligned}
& a_{l} k^{2}\left(2 n U_{l} U_{l}^{\prime \prime}+(1-2 n) U_{l}^{\prime 2}\right) \\
& \quad-4 n^{2}\left(a_{l} \kappa^{2}+\omega+\sum_{m=1}^{N} \lambda_{l m}\right) U_{l}^{2}+4 n^{2} b_{l} U_{l}^{3} \\
& \quad+4 n^{2} c_{l} U_{l}^{4}=0 .
\end{aligned}
$$

Balancing $U_{l} U_{l}^{\prime \prime}$ with $U_{l}^{4}$ in Eq. (259), then we get $s=4$. Using the solution procedure of the trial equation method, we obtain the system of algebraic equations as follows:

$U_{l}^{4}$ coeff.:

$$
a_{l} \alpha_{4} k^{2}(2 n+1)+4 n^{2} c_{l}=0,
$$

$U_{l}^{3}$ coeff.

$$
a_{l} \alpha_{3} k^{2}(n+1)+4 n^{2} b_{l}=0,
$$

$U_{l}^{2}$ coeff.:

$$
a_{l} a_{2} k^{2}-4 n^{2}\left(a_{l} \kappa^{2}+\omega+\sum_{m=1}^{N} \lambda_{l m}\right)=0,
$$

$U_{l}^{1}$ coeff.:

$$
-a_{l} \alpha_{1} k^{2}(n-1)=0,
$$

$U_{l}^{0}$ coeff.:

$$
a_{l} \alpha_{0} k^{2}(1-2 n)=0,
$$

Solving the above system of algebraic equations, we obtain the following results:

$$
\begin{aligned}
& \alpha_{0}=0, \quad \alpha_{1}=0, \\
& \alpha_{2}=\frac{4 n^{2}\left(a_{l} \kappa^{2}+\omega+\sum_{m=1}^{N} \lambda_{l m}\right)}{a_{l} k^{2}}, \\
& \alpha_{3}=-\frac{4 n^{2} b_{l}}{a_{l} k^{2}(n+1)}, \quad \alpha_{4}=-\frac{4 n^{2} c_{l}}{a_{l} k^{2}(2 n+1)}
\end{aligned}
$$

Substituting these results into Eqs. (4) and (5), we get

$$
\pm\left(\xi-\xi_{0}\right)=\int \frac{\mathrm{d} U_{l}}{\sqrt{\alpha_{2} U_{l}^{2}-\alpha_{3} U_{l}^{3}-\alpha_{4} U_{l}^{4}}} .
$$

Integrating (266) with respect to $U_{l}$, we obtain bright soliton solutions

$$
q^{(l)}(x, t)=\sqrt[2 n]{\frac{2(n+1)\left(a_{l} \kappa^{2}+\omega+\sum_{m=1}^{N} \lambda_{l m}\right)}{ \pm B_{l} \cosh \left(C_{l}\right)+b_{l}}} \mathrm{e}^{\mathrm{i} \Phi},
$$

This solution is valid for

$$
\begin{aligned}
& a_{l}\left(a_{l} \kappa^{2}+\omega\right)>0, \\
& 4(n+1)^{2} c_{l}\left(a_{l} \kappa^{2}+\omega\right)+(2 n+1) b_{l}^{2}>0 .
\end{aligned}
$$

The singular solitons are given by Eq. (270):

$$
q^{(l)}(x, t)=\sqrt[2 n]{\frac{2(n+1)\left(a_{l} \kappa^{2}+\omega+\sum_{m=1}^{N} \lambda_{l m}\right)}{ \pm B_{l} \sinh \left(C_{l}\right)+b_{1}}} \mathrm{e}^{\mathrm{i} \Phi},
$$


These are meaningful whenever

$$
a_{l}\left(a_{l} \kappa^{2}+\omega\right)>0,
$$

and

$$
4(n+1)^{2} c_{l}\left(a_{l} \kappa^{2}+\omega\right)+(2 n+1) b_{l}^{2}<0 .
$$

holds. In the above case we put:

$$
\begin{aligned}
& B_{l}=\sqrt{\frac{4 c_{l}(n+1)^{2}\left(a_{l} \kappa^{2}+\omega+\sum_{m=1}^{N} \lambda_{l m}\right)+b_{l}^{2}(2 n+1)}{2 n+1}}, \\
& C_{l}=\sqrt{\frac{4 n^{2}\left(a_{l} \kappa^{2}+\omega+\sum_{m=1}^{N} \lambda_{l m}\right)}{a_{l} k^{2}}\left(\xi-\xi_{0}\right) .}
\end{aligned}
$$

\section{Conclusions}

This paper studied solitons in nonlinear directional couplers with optical metamaterials. The integration scheme is the trial function method. Three types of couplers are taken into consideration. They are twincore couplers, multiple core couplers where coupling was with nearest neighbors and finally multiple-core couplers where coupling was with all neighbors. Each of these type of couplers were further handled with four forms of nonlinear media. They are Kerr law, power law, parabolic law and dual-power law. Thus, bright, dark and singular soliton solutions were retrieved. It must be noted that dark solitons solutions were recoverable only for the Kerr law nonlinearity for all of the three types of couplers. Such is the limitation of this approach.

The results of this paper carry a lot of scope for future studies. This scheme will be applied to other forms of nonlinear media such as anti-cubic nonlinearity. Additionally, this methodology shall be applied to other optical devices such as magneto-optic waveguides, DWDM systems, birefringent fibers, liquid crystals. Those results are awaited at this time. Moreover, the soliton solutions will be obtained in presence of several perturbation terms that are predominantly of Hamiltonian type. Therefore the readers are requested to patiently stay tuned.

\section{Acknowledgments}

The third author (SPM) would like to thank the research support provided by the Department of Mathematics and Statistics at Tshwane University of Technology and the support from the South African National Foundation under Grant Number 92052 IRF1202210126. The research work of sixth author (M.B.) was supported by Qatar National Research Fund (QNRF) under the grant number NPRP 8-028-1-001.

\section{References}

[1] A.A. Alshaery, E.M. Hilal, M.A. Banaja, S.A. Alkhateeb, L. Moraru, A. Biswas, J. Optoelectron. Adv. Mater. 16, 750 (2014).

[2] A.H. Arnous, M. Mirzazadeh, Q. Zhou, M.F. Mahmood, A. Biswas, M. Belic, Optoelectron. Adv. Mater. Rapid Commun. 9, 1214 (2013).

[3] A.H. Arnous, M. Mirzazadeh, S.P. Moshokoa, S. Medhekar, Q. Zhou, M.F. Mahmood, A. Biswas, M. Belic, J. Computat. Theor. Nanosci. 12, 5940 (2015).

[4] A.H. Arnous, M. Mirzazadeh, Q. Zhou, S.P. Moshokoa, A. Biswas, M. Belic, Optik 127, 11450 (2016).

[5] A.H. Arnous, M.Z. Ullah, S.P. Moshokoa, Q. Zhou, H. Triki, M. Mirzazadeh, A. Biswas, Optik 130, 996 (2017).

[6] A.H. Arnous, M.Z. Ullah, S.P. Moshokoa, Q. Zhou, H. Triki, M. Mirzazadeh, A. Biswas, Nonlin. Dyn. 88, 1891 (2017).

[7] A. Bahrami, A. Rostami, F. Nazari, Optik 122, 1787 (2011).

[8] A.Z. Chowdhury, Photonic Sensors 4, 34 (2014).

[9] C.-Q. Dai, Y.-Y. Wang, Nonlin. Dyn. 80, 715 (2015).

[10] M. Ekici, M. Mirzazadeh, Q. Zhou, S.P. Moshokoa, A. Biswas, M. Belic, Optik 127, 10879 (2016).

[11] M.M. El-Borai, H.M. El-Owaidy, H.M. Ahmed, A.H. Arnous, S.P. Moshokoa, A. Biswas, M. Belic, Optik 130, 324 (2017).

[12] X. He, Optik 125, 2267 (2014).

[13] M. Mirzazadeh, A.H. Arnous, M.F. Mahmood, E. Zerrad, A. Biswas, Nonlin. Dyn. 81, 277 (2015).

[14] M. Mirzazadeh, M. Ekici, Qin Zhou, A. Sonmezoglu, Superlatt. Microstruct. 101, 493 (2017).

[15] A.K. Sarma, Opt. Laser Technol. 41, 247 (2009).

[16] A.K. Sarma, Optik 120, 390 (2009).

[17] A.K. Sarma, Opt. Commun. 284, 186 (2011).

[18] M. Savescu, A.H. Bhrawy, A.A. Alshaery, E.M. Hilal, K.R. Khan, M.F. Mahmood, A. Biswas, J. Mod. Opt. 61, 441 (2014).

[19] J. Vega-Guzman, M.F. Mahmood, Q. Zhou, H. Triki, A.H. Arnous, A. Biswas, S.P. Moshokoa, M. Belic, Nonlin. Dyn. 87, 427 (2017).

[20] Y. Xiang, X. Dai, S. Wen, J. Guo, D. Fan, Phys. Rev. A 84, 033815 (2011). 